\title{
Some relationships between outcome expectancies and sample stimuli in pigeons' delayed matching
}

\author{
PETER J. URCUIOLI \\ Purdue University, West Lafayette, Indiana
}

\begin{abstract}
Two sets of experiments examined how differential outcomes affect conditional stimulus control by the samples in delayed matching-to-sample. Pigeons were initially trained on symbolic delayed matching with reinforcing outcomes that were either differential or nondifferential with respect to the samples. In one set of experiments, the outcome manipulation involved different ( $p=1.0$ vs. 0.2 ) versus the same ( $p=0.6$ ) probabilities of food; in the other, food and no-food outcomes were used. Following initial acquisition and mixed-delay tests, the matching procedure in each study was discontinued while the samples were nondifferentially reinforced with the same probability of food, or with food and no food, respectively. When later retested on delayed matching with those nondifferential outcomes, birds initially trained with different reinforcement probabilities matched at the same levels of accuracy as those trained with the same probability. By contrast, birds initially trained with food versus no-food outcomes showed lower levels of matching accuracy than their nondifferential controls. Subsequent transfer tests showed that matching performances by the differential birds in both studies had been originally cued in part by differential outcome expectancies. Apparently, the expectancies based upon different probabilities of food provided a source of conditional stimulus control that did not compete with the samples. By contrast, the expectation of food versus no food reduced (overshadowed) sample-stimulus control.
\end{abstract}

When the alternative correct responses in discrimination learning produce different reinforcing outcomes, acquisition and retention of the discrimination task are generally superior to that observed when all responses produce the same reinforcing outcomes (Carlson \& Wielkiewicz, 1976; Peterson, 1984; Trapold, 1970). For example, pigeons acquire matching-to-sample (MTS) and its variants more rapidly and show slower rates of forgetting when the correct response following each sample stimulus produces a distinctly different outcome (e.g., food vs. water; see Brodigan \& Peterson, 1976) than when outcomes either are the same on every trial or vary randomly across trials (DeLong \& Wasserman, 1981; Peterson, 1984; Peterson \& Trapold, 1982).

This differential outcomes effect (DOE) has been interpreted to mean that different reinforcing events provide a source of discriminative control in addition to whatever strengthening function they may have (e.g., Honig \& Dodd, 1986; Peterson, 1984; Trapold, 1970). According to this view, the correlation of different outcomes with the sample stimuli in MTS permits subjects to expect or anticipate early in a trial the particular outcome contingent upon a correct response later in the trial. Those out-

This research was supported by NSF Grant 86-06926. The author thanks Alice Golden, Nicholas Ketterer, Beth Kraemer, Judy Tedlie, and Suzanne Vanavermaete for running the subjects. Reprint requests should be addressed to the author, Department of Psychological Sciences, Purdue University, West Lafayette, IN 47907. come expectancies in turn cue which response to make. These points can be appreciated more readily by reference to Table 1. This table depicts both an actual and a hypothesized sequence of events occurring in MTS tasks involving differential, as opposed to nondifferential, outcomes. In both conditions, the sample stimuli $\left(S_{1}\right.$ and $\left.S_{2}\right)$ are associated with particular correct comparison-choice responses $\left(C_{1}\right.$ and $C_{2}$, respectively). Those responses produce distinctly different outcomes $\left(\mathrm{O}_{1}\right.$ and $\mathrm{O}_{2}$, respectively) in the differential condition, but each outcome is produced equally often in the nondifferential condition $\left(\mathrm{O}_{1} / \mathrm{O}_{2}\right)$. For the differential group, then, the samples can presumably give rise to different outcome expectancies ( $E_{1}$ and $E_{2}$, respectively), whereas for the nondifferential group, expectancies ought to be the same on each trial. The latter situation is represented here by the omission of $E_{1}$ and $E_{2}$ in order to indicate the lack of any additional differential stimulation.

Table 1

Diagram of the Events (Actual and Hypothesized) in Matching-toSample (MTS) with Differential Versus Nondifferential Outcomes

\begin{tabular}{cl}
\hline Outcomes & MTS Associations \\
\hline Differential & $\mathrm{S}_{1} \mathrm{E}_{1} \rightarrow \mathrm{C}_{1}\left(\mathrm{O}_{1}\right)$ \\
& $\mathrm{S}_{2} \mathrm{E}_{2} \rightarrow \mathrm{C}_{2}\left(\mathrm{O}_{2}\right)$ \\
& $\mathrm{S}_{1} \rightarrow \mathrm{C}_{1}\left(\mathrm{O}_{1} / \mathrm{O}_{2}\right)$ \\
Nondifferential & $\mathrm{S}_{2} \rightarrow \mathrm{C}_{2}\left(\mathrm{O}_{1} / \mathrm{O}_{2}\right)$ \\
\hline
\end{tabular}


The view that differential outcome expectancies have discriminative properties is also supported by two other findings in the literature. First, reversing or eliminating differential outcomes following initial training typically causes otherwise accurate performances to deteriorate (Honig, Matheson, \& Dodd, 1984; Peterson, Wheeler, \& Armstrong, 1978). Such an effect is understandable assuming that those manipulations alter one of the functional cues (viz, the expectancy cue) governing performance. Second, MTS performance with differential outcomes readily transfers to novel stimuli that have the same outcome associates as the samples used in training (Edwards, Jagielo, Zentall, \& Hogan, 1982; Peterson, 1984). In a particularly compelling demonstration of the latter effect, Peterson (1984) trained pigeons on symbolic delayed MTS with red and green samples and food versus tone outcomes, and also provided concurrent, off-baseline training in which two geometric forms were correlated with the same two outcomes. When the forms were later substituted for their corresponding hue-sample associates in the matching task, Peterson found immediate and strong positive transfer of performance. This result is explicable assuming that the outcome expectancies associated with the forms were the same as, or similar to, those providing a discriminative cue for choice in the matching task (cf. Edwards et al., 1982).

Although these results provide good evidence for the discriminative properties of outcome expectancies, they do not completely clarify how those properties, or the differential outcomes that give rise to them, contribute to or produce the DOE. For example, it is unclear what, if any, effect differential outcomes have on conditional stimulus control by the samples. One possibility is that they enhance sample-stimulus control. As Peterson and Trapold (1982) noted,

differential outcomes procedure facilitates conditional discrimination learning by enhancing the difference between the functional conditional cues ... Thus, the DOE is .. a special case of acquired stimulus distinctiveness. (p. 572)

Edwards et al. (1982) made a similar point, stating that

differential expectancies can enhance the discriminability of two stimuli to the extent that those stimuli are difficult to discriminate. (p. 257)

Thus, the DOE could arise because sample-comparison associations develop more rapidly and/or are stronger in groups trained with differential, as opposed to nondifferential, outcomes.

Another possibility is that differential outcomes simply add another source of conditional stimulus control without affecting concurrent control exerted by the samples. Consequently, learning may be more rapid with differential outcomes than with nondifferential outcomes because subjects in the former condition have two discriminative cues upon which to base their performances instead of only one, as implied in Table 1 .

Finally, the DOE may occur because outcome expectancies are simply more salient (and hence more dominant in the control over choice) than the sample stimuli. Although this view is similar to the alternative just described (i.e., two cues are better than one), the suggestion here is that the redundancy and salience of differential outcome expectancies may also reduce (overshadow) the conditional stimulus control otherwise acquired by the samples. In a comment on a study by Peterson and Trapold (1980), Honig et al. (1984) raised this point when they noted that

expectancies ... overshadowed any residual direct control of responding by the initial stimuli. (p. 198)

The overshadowing alternative is particularly intriguing because the same sort of competing interaction has been found to occur between sample stimuli and differential sample behaviors in MTS. Specifically, the amount of control the samples normally acquire over pigeons' matching performances is reduced if the birds must respond differentially to the samples in order to produce the comparisons (Urcuioli, 1984; see also Urcuioli, 1985). Perhaps outcome expectancies also act in this fashion. After all, they produce other effects like those seen with differential sample responding. For example, they provide a source of conditional stimulus control independent of the original samples with which they were associated (Peterson, 1984; cf. Urcuioli \& Honig, 1980). Also, they facilitate acquisition to the extent that they provide a valid cue for later responding (Honig et al., 1984; Peterson \& Trapold, 1980; cf. Cohen, Looney, Brady, \& Aucella, 1976; Urcuioli, 1985).

The possibility of overshadowing is also directly suggested by the finding that on-baseline shifts from differential to nondifferential outcomes severely disrupt pigeons' delayed matching performances (Peterson et al., 1978; Peterson, Wheeler, \& Trapold, 1980). Thus, the samples alone appear to be unable to maintain high levels of accuracy when the outcome expectancies ostensibly present during acquisition are removed.

Although the on-baseline-shift results currently represent the best evidence for overshadowing in differential outcomes paradigms, the results do not force that interpretation. For instance, matching accuracy may have deteriorated because the shifts produced a surprising change in trial outcomes and/or because the nature of one of the cues controlling performance (viz, the expectancy cue) was changing. Furthermore, it is not clear from this previous work whether the "residual direct control" by the samples was actually less than what it would have been had no redundant expectancy cue been present during initial acquisition (Mackintosh, 1974).

A better test of residual direct control is to remove the expectancy cue prior to testing and then to compare matching accuracy with that shown by a comparably treated control group. Removing the expectancy cue can presumably be accomplished by changing the relationship between samples and outcomes outside of the matching context. After all, Peterson (1984) showed that stimuli associated off-baseline with the same differential outcomes as the samples readily substitute for the latter. By implication, then, off-baseline changes in sample-outcome associations 
should likewise affect outcome expectancies. If so, then that technique would permit a more accurate assessment of conditional stimulus control by the samples by minimizing or avoiding the interpretive complications of the onbaseline procedure mentioned above.

In the two sets of experiments reported in this paper, the off-baseline manipulation was used to evaluate the three explanations of the DOE by comparing samplestimulus control in differentially versus nondifferentially trained groups. Specifically, these experiments examined whether such control was stronger, weaker, or no different following differential outcomes training than following nondifferential training. The experimental approach was to (1) initially demonstrate a DOE with the matching procedures used here, (2) subsequently assess the degree to which the samples alone controlled performances in each group by first "extinguishing" any outcome-expectancy cue outside of the matching context, and (3) show that differential outcome expectancies had indeed provided a discriminative cue for the differentially trained subjects.

In one set of experiments, the outcomes were the same versus different probabilities of food reinforcement (cf. Santi \& Roberts, 1985). In a second set, the outcomes were food and no food scheduled equally often, or differentially, on the two sample trial types. The latter experiments were run subsequent to the first in order to clarify some ambiguities in the interpretation of the results from the probability study. However, the corresponding experiments from each study are described concurrently in this paper and are labeled " $A$ " and " $B$ " in order to make across-study comparisons easier.

\section{EXPERIMENTS 1A AND 1B}

The first experiment of each study compared rates of MTS acquisition and subsequent retention performances as a function of whether the outcomes for correct comparison choice were differential or nondifferential with respect to the samples. The same two-alternative matching task was used in both experiments: Pigeons matched line-orientation samples to hue comparison stimuli. The type of reinforcing outcomes, however, differed across experiments. In Experiment $1 \mathrm{~A}$, the probability of food reinforcement was manipulated across conditions: for the differential group, different probabilities of food were associated with each sample; for the nondifferential group, the probability of food following correct choice was the same on all trials. In Experiment 1B, food and no-food outcomes were scheduled after correct choices following the two samples in the differential group, and each outcome was scheduled equally often following all correct choices in the nondifferential group.

These experiments provided preliminary data regarding the possible contribution of an outcome-expectancy cue to matching performances (i.e., Experiments $1 \mathrm{~A}$ and $1 \mathrm{~B}$ tested for a DOE). Additionally, they provided a baseline against which to judge the effects, if any, of the sub- sequent off-baseline manipulation of the sample-outcome associations. Finally, by varying the type of outcomes across experiments, a rough assessment was made of the relative salience of different probabilities of food versus the presence versus absence of food. The working hypothesis was that an outcome-expectancy cue associated with food versus no food would be more salient than that associated with different, nonzero probabilities of the same event. If so, the DOE should be larger in Experiment 1B than in Experiment 1A.

\section{Method}

Subjects. The subjects in each experiment were 16 experimentally naive White Carneaux pigeons. All birds were retired breeders obtained from the Palmetto Pigeon Plant (Sumter, SC). The subjects were housed individually in wire-mesh cages in a colony room with a 14:10-h light:dark cycle. Immediately prior to the experiments, birds were food-deprived to $80 \%$ of their free-feeding body weights as established by unrestricted access to grain in the home cages for a period of not less than 5 days. The birds were maintained at their $80 \%$ levels by daily adjustment in the reinforcement period for each experimental session. Supplemental feedings were generally given only on the 1 day/week that the birds were not run. Water and grit were continuously available in the home cage.

Both Experiments $1 \mathrm{~A}$ and $\mathrm{BB}$ (and the corresponding ones that followed them) were run in two replications, with 8 subjects in each. Data from both replications of each experiment have been combined for presentation and analysis because the results were similar. Three of the original subjects trained with food and no-food outcomes were replaced by other experimentally naive birds at various points during that study. One subject failed to acquire MTS after 60 sessions of training, another could not maintain accurate performance at the 0 -sec training value during the mixed-delay retention tests immediately following acquisition, and the third showed evidence that its performance during the subsequent nondifferential tests was influenced by differential sample behaviors (Urcuioli \& Honig, 1980)

Apparatus. The experiments were run in a single BRS/LVE pigeon chamber, containing a three-key intelligence panel (Model PIP-016) with inline stimulus projectors (Model IC-901-IDD). The pigeon's compartment within the chamber measured $37 \times 30 \times 34 \mathrm{~cm}$ (height $\times$ width $\times$ depth). Three circular response keys, $2.5 \mathrm{~cm}$ in diameter and spaced $8.3 \mathrm{~cm}$ center to center, were mounted in a row $10 \mathrm{~cm}$ from the top of the intelligence panel. Stimulus projectors mounted behind each key could project three vertical (V) or horizontal $(\mathrm{H})$ black lines on a white background, as well as white $(\mathrm{W})$, red (R), and green (G) homogeneous fields (Pattern No. 715). Centered approximately $13 \mathrm{~cm}$ below the center key was a $5 \times$ $5.8 \mathrm{~cm}$ opening for the rear-mounted food magazine. Operation of the food magazine was modified for Experiment 1B so that activation of the solenoid that raised the hopper was independent of illumination of the stimulus bulb (24-ESB) inside the magazine housing. General chamber illumination was provided by a partially covered houselight (General Electric No. 1829 bulb) that directed light toward the ceiling. The chamber was ventilated by a blower fan mounted on the outside of the apparatus, which also helped to mask extraneous noises. All experimental events were controlled and recorded by an IBM-PCXT located in an adjacent room.

Procedure. Following magazine training, all birds were shaped by the method of successive approximations to peck a $W$ stimulus on the center response key. They then received one 60 -trial session during which single keypecks to $\mathrm{V}$ and $\mathrm{H}$ center-key lines were reinforced. This was followed on the next day by another 60 -trial session during which center-key pecks to $\mathrm{V}$ and $\mathrm{H}$ and side-key pecks to $R$ and $G$ were reinforced. In both of these preliminary 
training sessions, the line and hue stimuli appeared an equal number of times on their respective keys, with successive stimulus presentations separated by a 10 -sec intertrial interval (ITI). A single peck to the lighted key on any trial produced 3 -sec access to a lighted food hopper.

Pretraining. Next, all birds received additional training (referred to as pretraining) during which presentations of the $\mathrm{V}$ and $\mathrm{H}$ centerkey lines were followed by either the same or the different outcomes. In Experiment 1A, a single peck to the line stimulus for each trial initiated a fixed-interval (FI) schedule. After the FI, the first peck turned off the line and produced food reinforcement with a probability determined by group assignment. For the birds in Group Diff, reinforcement occurred after every presentation of one orientation ( $p=1.0$ ), but reinforcement occurred after only $20 \%$ of the trials with the other orientation ( $p=0.2)$, counterbalanced across subjects. For the birds in Group Nondiff, reinforcement $o c-$ curred on $60 \%$ of the trials with each stimulus $(p=0.6)$. The FI value was $2 \mathrm{sec}$ for the first two sessions of pretraining and $3 \mathrm{sec}$ for the next three.

In Experiment 1B, the line stimulus on each pretraining trial was followed after $3 \mathrm{sec}$ by either the lighted food hopper (food outcome) or illumination of the hopper light alone (no-food outcome) independently of responding. For the birds assigned to Group Diff, the food outcome consistently occurred following offset of one line and the no-food outcome consistently occurred following offset of the other, counterbalanced across subjects. For the birds in Group Nondiff, the food and no-food outcomes occurred equally often following each stimulus. For all subjects in Experiment 1B, pretraining lasted for three sessions.

In both experiments, the $\mathrm{V}$ and $\mathrm{H}$ lines each appeared 30 times and in random order in each pretraining session, with the constraint that neither stimulus could appear on more than three successive trials. Additionally, each trial began with the presentation of a W center-key "ready" stimulus. The first peck to the W stimulus immediately turned it off and, 500 msec later, produced one of the two lines on the center key. Successive trials were again separated by a 10-sec ITI. The houselight was off for the first $9 \mathrm{sec}$ of the ITI but was turned on $1 \mathrm{sec}$ prior to the presentation of the $W$ "ready" stimulus. It then remained on until the end of the trial, which was defined as the end of the reinforcement cycle or the equivalent period following stimulus offset. The duration of food presentation was adjusted daily for individual birds in order to maintain their body weights at around the $80 \%$ level (range: $2-6 \mathrm{sec}$ ). The durations of the nonreinforcement period in Experiment $1 \mathrm{~A}$ and of the no-food event in Experiment 1B were the same as that for reinforcement in each session.

Symbolic MTS acquisition and retention. Following pretraining, the birds began training on 0-delay symbolic MTS with $V$ and $H$ sample stimuli, $R$ and $G$ comparisons, and differential or nondifferential outcomes for correct comparison choices. In each experiment, MTS sessions consisted of 80 trials, 40 with each sample stimulus. All trials began with the illumination of the houselight $1 \mathrm{sec}$ prior to the onset of a W center-key "ready" stimulus. A peck to the W center key turned this stimulus off and, $500 \mathrm{msec}$ later, produced either the $\mathrm{V}$ or $\mathrm{H}$ sample on the same key. In Experiment $1 \mathrm{~A}$, the first peck to $\mathrm{V}$ or $\mathrm{H}$ initiated a 3 -sec sampleobservation period; the first peck after this FI turned the sample off and immediately produced the $R$ and $G$ comparisons on the adjacent side keys. In Experiment IB, the corresponding 3-sec period began as soon as the sample appeared and ended automatically after $3 \mathrm{sec}$; in other words, the presentation of the comparisons in this experiment was response-independent.

In both experiments, a single peck to either comparison hue turned both stimuli off and produced the programmed reinforcing outcome if the choice was "correct" or an equivalent timeout period with the houselight turned off if the choice was "incorrect." For half of the birds in each outcome condition of each experiment, an $\mathbf{R}$ choice was correct after the $V$ sample and a $G$ choice was correct after $\mathrm{H}$; for the remaining birds, these relations were reversed. Additionally, for the birds in Group Diff of Experiment 1A, correct choices were reinforced by food with a probability of 1.0 following one sample and a probability of 0.2 following the other. For the corresponding birds in Group Nondiff, all correct choices were reinforced by food with a probability of 0.6 . The assignment of probability values to samples in both groups was identical to that used during pretraining. Correct but nonreinforced trials ended with the comparisons turned off but with the houselight remaining on for a period equivalent to the reinforcement cycle

In Experiment 1B, correct choices for the birds in Group Diff always produced food following one sample and no-food following the other, with sample-outcome relations counterbalanced across subjects. For the birds in Group Nondiff, correct choices on all trials were followed equally often by each outcome. Incorrect choices in this experiment resulted in the repetition of the same trial (i.e., a correction procedure was used), although only performance on the first trial was used to compute matching accuracy. The reason for using a correction procedure was to avoid the possibility that the Group Diff birds might simply respond through no-food trials (e.g., adopt a position habit) in order to advance to subsequent foodreinforced trials.

In both experiments, the four possible sample-comparison configurations were presented five times in each successive block of 20 trials. Trials were separated by a $10-\mathrm{sec}$ ITI, the first $9 \mathrm{sec}$ of which were spent in darkness. The durations of food reinforcement, no food (if applicable), and timeout were identical in a given session, although this value again varied from $2-5 \mathrm{sec}$ across sessions in order to maintain each bird's $80 \%$ body weight. Individual birds were run on their respective tasks for a minimum of 10 sessions and until they chose the correct comparison on $90 \%$ of all trials on five of six consecutive sessions.

Following acquisition, the birds were run for 15 additional sessions with delays of $0,1,2$, and $4 \mathrm{sec}$ separating sample offset and comparison onset. The four delay values occurred equally often with each sample-comparison configuration (i.e., 20 times) in each mixed-delay session. All other procedural details, however, were the same as during acquisition.

The Type I error rate for all statistical analyses reported in this paper was .05 , as defined by Rodger's (1975) critical $F$ values.

\section{Results and Discussion}

Figure 1 shows symbolic MTS acquisition for the two groups in each experiment over the first 10 sessions of training. The top panel presents the acquisition data from Experiment 1A; the bottom panel presents the corresponding data from Experiment 1B. A DOE is clearly evident in both experiments: Matching accuracy increased more rapidly over sessions in Groups Diff than in Groups Nondiff. This was confirmed by an analysis of variance (ANOVA), which showed a significant between-group effect in both Experiments $1 \mathrm{~A}$ and $1 \mathrm{~B}\left[F_{\mathrm{S}}(1,14)=6.62\right.$ and 33.51 , respectively] and significant group $\times$ session interactions $[F \mathrm{~s}(9,126)=3.66$ and 27.18$]$. The improvement in performance over sessions averaged across the groups in each experiment was also significant $[F \mathrm{~s}(9,126)=$ 23.74 and 53.18, for Experiments $1 \mathrm{~A}$ and $1 \mathrm{~B}$, respectively].

Figure 2 shows the retention functions for these groups averaged over the last 10 sessions of mixed-delay testing. ${ }^{1}$ Overall accuracy was higher and retention loss was slower in Group Diff than in Group Nondiff in each 

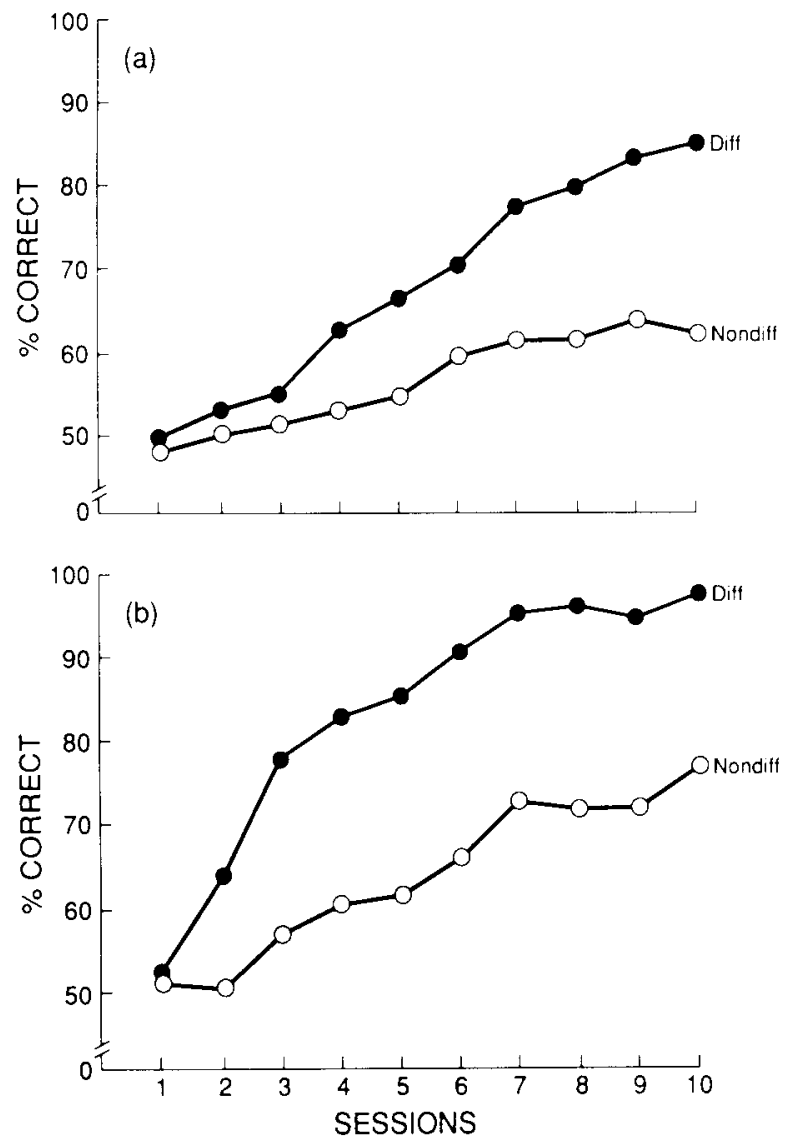

Figure 1. The average percentage of correct choice responses for the two groups in Experiment 1A (top) and Experiment 1B (bottom) over the first 10 sessions of symbolic MTS acquisition.

experiment. Indeed, in Experiment 1B (bottom panel), Group Diff showed essentially no loss in matching accuracy up to the longest delay value tested $(4 \mathrm{sec})$. ANOVAs confirmed the between-group differences evident in Figure 2. The overall level of performance in Group Diff was more accurate than in Group Nondiff $[F(1,12)=29.88$, and $F(1,13)=70.65$, Experiments $1 \mathrm{~A}$ and $1 \mathrm{~B}$, respectively]. Additionally, the group $\times$ delay interactions were significant $[F(3,36)=12.23$, and $F(3,39)=45.54$, respectively], as were the overall delay effects $[F(3,36)=155.05$ and $F(3,39)=51.51$, respectively].

The enhancement produced by differential outcomes also appeared to be larger when those outcomes were food versus no food than when they were different probabilities of the same food reinforcer. For example, in Experiment $1 \mathrm{~B}$, the average number of sessions needed by Group Diff and Group Nondiff to reach $90 \%$ or better accuracy during MTS acquisition was 5.2 and 22.8, respectively. The figures for the corresponding groups in Experiment $1 \mathrm{~A}$ were 11.4 and 23.8. Thus, although the nondifferentially trained birds in each experiment acquired at roughly the same rate, the Group Diff birds did not. Those trained with food versus no food learned faster than did those trained with different probabilities of food.
Additionally, the difference in the DOEs was apparent during subsequent retention testing with mixed delays, as shown by the more widely separated retention functions in Experiment 1B than in Experiment 1A.

These experiments demonstrate, then, that symbolic MTS acquisition and retention are enhanced when different reinforcing outcomes are associated with each sample (and its correct comparison) than when the same outcome occurs equally often following each trial type. Neither finding is novel (see, e.g., Peterson, 1984; Santi \& Roberts, 1985), but each is important because it provides preliminary evidence that choices by the Group Diff birds were controlled at least in part by differential outcome expectancies (see Table 1). Furthermore, the acquisition and retention results are consistent with the supposition that the expectation of food versus no-food outcomes provided a more salient cue for choice than did the expectation of different probabilities of food reinforcement.

\section{EXPERIMENTS 2A AND 2B}

The next experiment in each study tested the degree to which the samples alone controlled matching perfor-
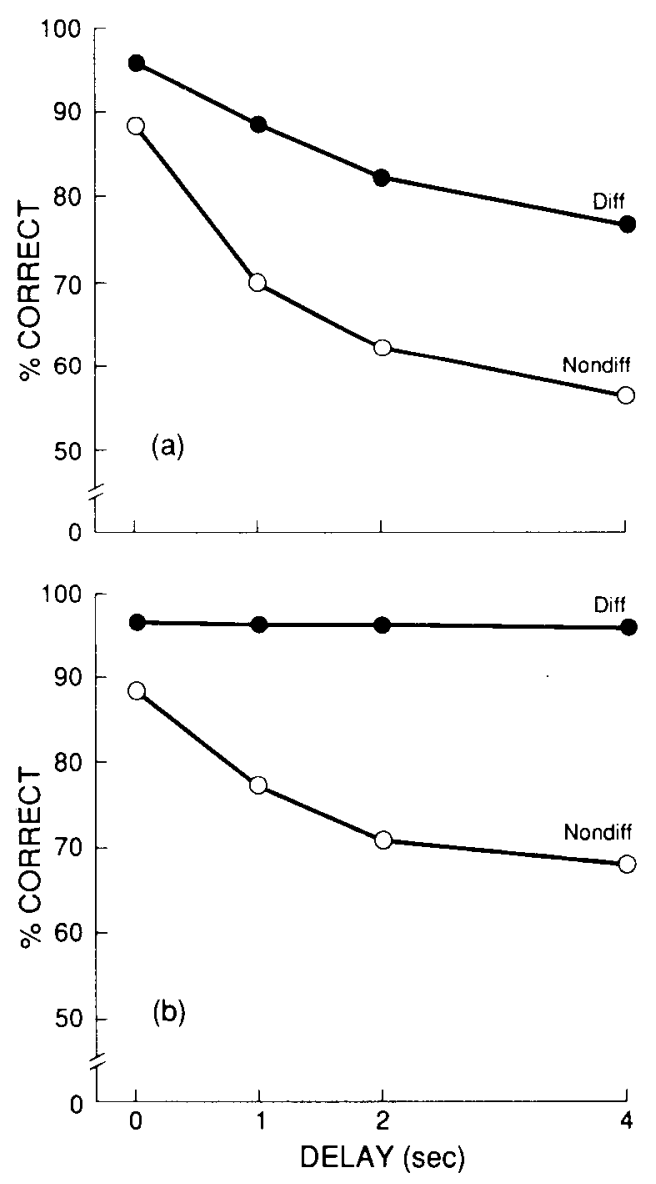

Figure 2. Retention gradients averaged over the last 10 mixeddelay sessions for the two groups in Experiment $1 \mathrm{~A}$ (top) and Experiment $1 B$ (bottom). 
mances by the differentially versus nondifferentially trained subjects. Of particular interest was how well the Group Diff birds would continue to match after their presumed outcome-expectancy cue was removed. Would they match at the same level as the Group Nondiff birds during testing or would their performances be less (or perhaps more) accurate?

For instance, if the development of sample-comparison associations during initial acquisition and testing was enhanced by differential outcomes (e.g., by making the samples and/or the comparisons more distinctive), then the stronger associations should be revealed by higher levels of matching accuracy in Groups Diff than in Groups Nondiff once the "masking" effect of the outcome-expectancy cue is removed. Alternatively, if differential outcome expectancies overshadowed the samples, Groups Diff should perform more poorly in testing than Groups Nondiff. Finally, if the expectancy cue functioned independently of the samples, then the two groups ought to perform similarly in testing.

To conduct these tests, the birds were initially returned to the pretraining phase. Now, however, the reinforcing events following each center-key (sample) stimulus were nondifferential with respect to those samples for all subjects. Afterwards, the symbolic MTS task was reintroduced but with nondifferential outcomes following all correct choices in both groups. Matching accuracy during nondifferential testing was measured at both the training value alone $(0 \mathrm{sec})$ and, subsequently, at the various delays programmed in the mixed-delay sequence (cf. Experiments $1 \mathrm{~A}$ and $1 \mathrm{~B}$ ).

\section{Method}

Subjects and Apparatus. The subjects and apparatus used in Experiments 2A and 2B were identical to those used in Experiments $1 \mathrm{~A}$ and $\mathbf{1 B}$.

Procedure. After their last mixed-delay session, all birds from Experiment 1A (i.e., those trained with the same or different probabilities of food) received one to three refresher sessions on their MTS acquisition task to ensure high levels of 0-delay matching accuracy. Similar refresher training was not provided for the birds from Experiment 1B (i.e., those trained with food and no-food outcomes)

Next, the MTS task was temporarily discontinued for all birds while they received nondifferential reinforcement following each center-key (sample) stimulus. These off-baseline sessions were identical to those described for the Group Nondiff birds in the preceding experiments. In Experiment $2 \mathrm{~A}$, all birds received 10 sessions of off-baseline nondifferential pretraining. The probability of food reinforcement following each center-key (sample) stimulus during the first five of these sessions was 1.0 for both the Group Diff and Group Nondiff birds, in order to make the change from differential to nondifferential outcomes for the former as distinctive as possible and to partially control for the effects of change per se. For the last five pretraining sessions, the probability was reduced to 0.6 in both groups.

In Experiment 2B, each center-key (sample) stimulus was now followed equally often by presentations of food and no-food in both groups. The subjects in this experiment received different numbers of off-baseline sessions. Specifically, each bird in Group Diff received as many pretraining sessions as necessary so that their behavior in the presence of the line stimuli appeared to be nondifferen- tial. This meant that any pecks to the $\mathrm{V}$ and $\mathrm{H}$ lines occurred about equally often to each stimulus and/or that the average latencies to make the first peck to the lines were approximately equal. Between 3-12 pretraining sessions were required to accomplish this. Each bird in Group Nondiff was matched with a Group Diff partner during the off-baseline phase in order to equate for the average number of these sessions across groups.

Next, all birds in both experiments were returned to 0-delay symbolic MTS with nondifferential reinforcement for correct comparison choice. In other words, all correct choices for both groups of Experiment $2 \mathrm{~A}$ were reinforced by food with a probability of 0.6 . Similarly, in Experiment 2B, food and no-food outcomes followed all correct comparison choices equally often. All other aspects of the matching procedure, however, were identical to those described for the corresponding MTS tasks used in Experiments $1 \mathrm{~A}$ and $1 \mathrm{~B}$. Each bird was given a minimum of three sessions during this initial nondifferential test and was given additional sessions (if necessary) until it matched correctly on $90 \%$ or more trials in a single session. Once this level of performance was achieved, each bird received 10 additional sessions of mixed-delay tests with nondifferential outcomes

\section{Results}

Figure 3 presents the average performance for the two groups in each experiment on the first three nondifferential test sessions. Also shown is accuracy averaged over the last five baseline (B) acquisition sessions in Experi-
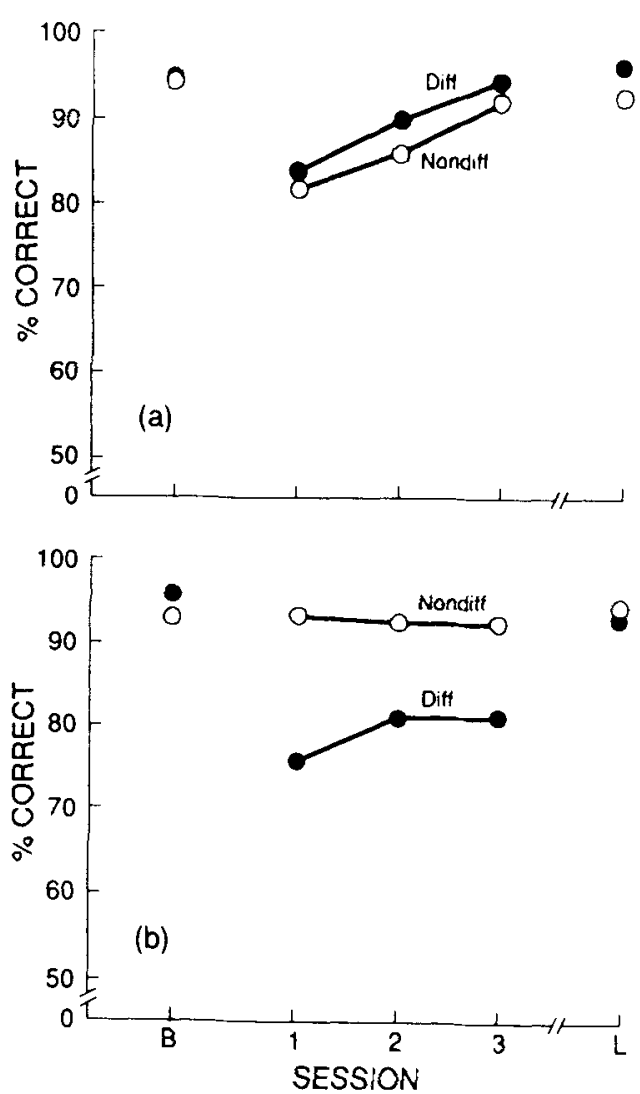

Figure 3. Nondifferential test performances for each group on the first three and last (L) 0 -delay test session in Experiment $2 \mathrm{~A}$ (top) and Experiment 2B (bottom). Corresponding performance averaged over the last five baseline (B) acquisition sessions in Experiments $1 A$ and $1 B$ are also shown. 
ments $1 \mathrm{~A}$ and $1 \mathrm{~B}$ and accuracy averaged over the last nondifferential test session (L) for each bird in each group.

Performances during nondifferential testing in Experiment 2A (top panel) did not differ appreciably between groups. Although the percentage of correct choices on the initial test sessions dropped relative to baseline, the size of that drop was similar for the two groups (10.9\% and $12.1 \%$ for Group Diff and Group Nondiff, respectively). An ANOVA on data for the first three test sessions revealed that only the sessions effect was significant $[F(2,28)$ $=19.47$ ]. Neither the effect of group nor the group $X$ session interaction were significant $(F \mathrm{~s}<.30)$. Not surprisingly, accuracy computed on the basis of each bird's last test session (L) also did not differ between groups $[F(1,14)=2.96]$.

By contrast, initial test performances in Experiment 2B (bottom panel) did differ substantially between groups. In particular, the shift to nondifferential outcomes for Group Diff produced a large drop in its matching accuracy relative to baseline, an effect not seen following the corresponding off-baseline sessions for Group Nondiff. Consequently, performance by Group Diff during the initial three test sessions was less accurate than that by Group Nondiff.
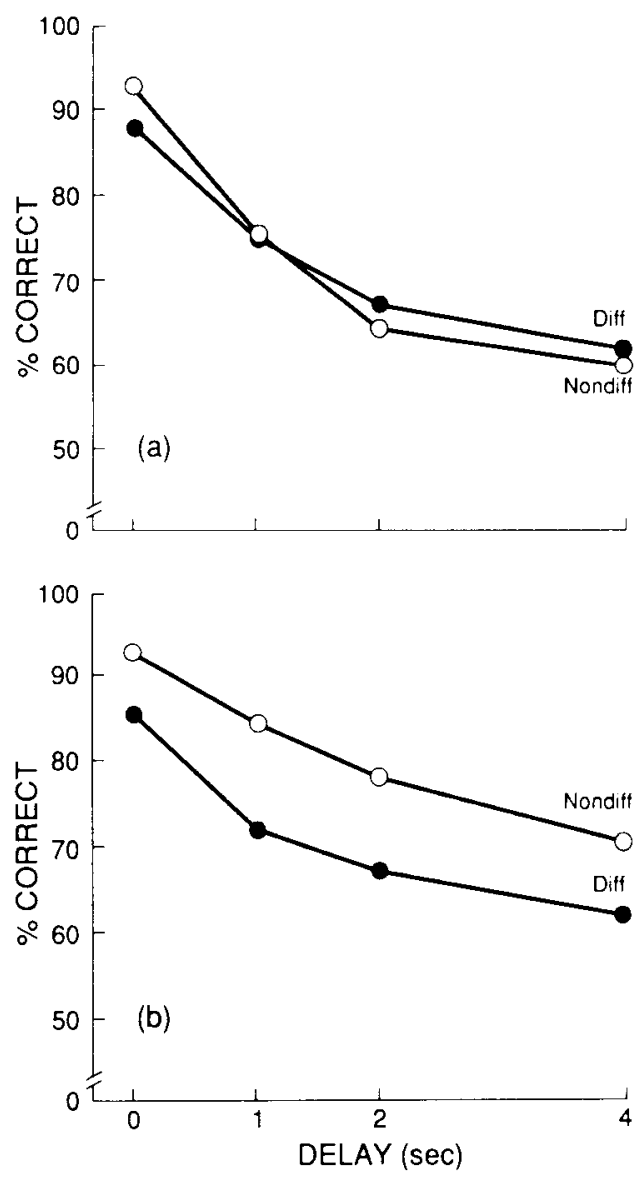

Figure 4. Retention gradients for each group averaged over the 10 mixed-delay sessions with nondifferential reinforcement in Experiment $2 \mathrm{~A}$ (top) and Experiment 2B (bottom).
An ANOVA on these data confirmed that Group Diff performed less accurately overall than did Group Nondiff $[F(1,14)=26.30]$. However, neither the sessions effect nor the group $\times$ session interaction were significant $[F \mathrm{~s}(2,28)=0.96$ and 1.95$]$. Additional one-way ANOVAs on the performances for each of the first three test sessions revealed that the between-group difference was significant on all of them $[F \mathrm{~s}(1,14)=47.37,8.11$, and 11.46 , respectively]. By the end of each bird's nondifferential tests $(L)$, however, accuracy of performance for the two groups did not differ significantly $[F(1,14)=$ 2.48], although Group Diff needed more sessions on average to reach $90 \%$ accuracy than Group Nondiff (5.5 vs. 1.8 , respectively) $[F(1,14)=13.24]$.

Figure 4 shows the retention gradients for these groups averaged over the 10 mixed-delay sessions that followed the 0-delay tests. In Experiment 2A (top panel), the retention functions for the two groups were virtually identical. Thus, the similar performances seen when nondifferential tests were run with only 0 -sec delays (see top panel of Figure 3) were also obtained when longer delays separated sample offset from comparison onset. An ANOVA on the retention data from this experiment showed no between-group difference in accuracy averaged across delays $[F(1,12)=.01]$, although the effect of delay averaged across groups was significant $[F(3,36)$ $=135.76$. The group $\times$ delay interaction was also significant $[F(3,36)=2.23]$. However, one-way ANOVAs on the performances at each delay failed to reveal a significant between-group difference at any of them [all $\left.F_{\mathrm{s}}(1,12)<1.60\right]$.

The retention results from Experiment 2B (bottom panel) were quite different: Matching was less accurate at every delay in Group Diff than in Group Nondiff. This result was obtained in spite of the fact that nondifferential test performances immediately prior to the second retention test were comparable (see L points in the bottom panel of Figure 3). The reemergence of between-group differences when mixed delays were reintroduced was confirmed by analysis of variance, which showed a significant between-group effect $[F(1,13)=11.26]$. The effect of delay was also significant $[F(3,39)=96.41]$, but the group $\times$ delay interaction was not $[F(3,39)=1.13]$.

Figure 5 summarizes the changes in performance from the first to the second mixed-delay test for the two groups in each experiment. Matching accuracy by delay averaged over the last 10 mixed-delay sessions of Experiments $1 \mathrm{~A}$ and $1 \mathrm{~B}$ (Test 1) is plotted along with the data from the 10 mixed-delay sessions in this experiment (Test 2). Note that the data labeled "Test 1" reproduce the functions shown in Figure 2, and those labeled "Test 2" reproduce the functions in Figure 4.

The shift from differential to nondifferential outcomes in Group Diff of Experiment 2A (top panel, left) produced a large drop in its delayed-matching accuracy. By contrast, the second nondifferential retention test for Group Nondiff (top panel, right) yielded a slight improvement in its memory performance. ANOVAs on the data from 
Group Diff confirmed that overall levels of delayedmatching accuracy dropped from Test 1 to Test $2[F(1,12)$ $=8.87]$. The overall effect of delay was also significant $[F(3,36)=100.64]$, as was the interaction of test $\times$ delay $[F(3,36)=3.63]$. For Group Nondiff, overall levels of performance did not differ between tests $[F(1,12)=$ 1.82], nor was there any interaction between test and delay $[F(3,36)=.57]$. Only the effect of delay averaged across tests was significant $[F(3,36)=188.26]$.

An ANOVA on retention data of Group Diff from Experiment 2B (bottom panel, left) showed that accuracy on the nondifferential test (Test 2) was significantly lower than on the prior differential test (Test 1$)[F(1,7)=$ 95.95]. The effect of delay averaged over tests was also significant, as was the test $\times$ delay interaction $[F \mathrm{~s}(3,21)$ $=45.41$ and 45.48 , respectively]. For Group Nondiff (bottom panel, right), overall retention accuracy improved from the first to the second nondifferential test $[F(1,6)=$ 15.69]. The delay effect and the test $\times$ delay interaction were significant as well $[F \mathrm{~s}(3,18)=67.92$ and 2.90 , respectively].

\section{Discussion}

The results of the nondifferential tests in these two experiments provided different answers to the question of how well sample stimuli control pigeons' matching performances after differential outcomes training. The results of Experiment $2 \mathrm{~A}$ showed that, in the absence of differential outcomes (and the expectancy cue to which they presumably give rise), the line samples exerted no less control over choice than they did when they provided the only cue for choice throughout initial training. Furthermore, conditional stimulus control by the samples was also no stronger in the former than in the latter condition. These results are contrary to both an overshadowing and an enhancement interpretation of how differential outcomes influence sample-stimulus control in MTS. Had outcome expectancies overshadowed the samples, matching performances in Group Diff in the absence of the expectancy cue should have been less accurate than those in Group Nondiff. Conversely, if attention to the samples had been enhanced by prior differential outcomes training (i.e., if sample-comparison associations were strengthened by such training), then the opposite pattern of results would be anticipated. Instead, the finding that Group Diff performed similarly to Group Nondiff during the nondifferential tests of Experiment $2 \mathrm{~A}$ suggests that the outcome-expectancy cue associated with different reinforcement probabilities had simply provided the former birds with an additional cue for choice. Removing that cue caused performance to drop to the same level as that maintained by the samples alone in Group Nondiff.

It is important to emphasize the fact that Group Diff performed at the same level of accuracy as Group Nondiff not only during the 0-delay tests but also during the subsequent mixed-delay tests. One could argue, for example, that since the DOE itself is sometimes small and unreliable at very short retention intervals (Brodigan \& Peterson, 1976; DeLong \& Wasserman, 1981; Edwards et al., 1982; Peterson et al., 1980), the finding that the groups did not differ in their 0-delay performances during initial nondifferential testing is not particularly sig-

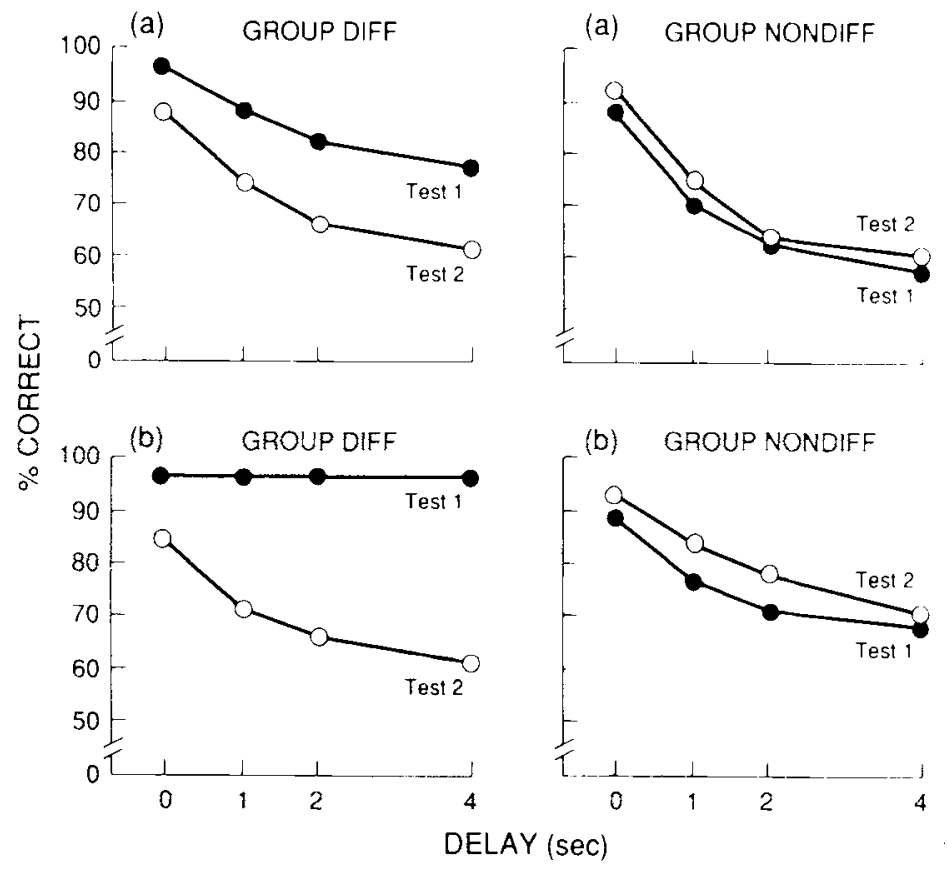

Figure 5. Retention gradients from the first and second mixed-delay tests given to each group in Experiments 1A and 2A (top) and in Experiments $1 B$ and 2B (bottom). 
nificant. But given that the DOE is very reliable and often quite pronounced at relatively long retention intervals (suggesting perhaps that outcome expectancies exert stronger control with longer delays), then the finding that there was also no between-group difference in the strength of sample-stimulus control at these longer delays is noteworthy.

Of course, the results of Experiment 2A by themselves might be dismissed on the grounds that the method of testing was simply insensitive to detecting between-group differences in performances. However, that argument is dispelled by the results of Experiment 2B, which revealed substantial between-group differences using essentially the same off-baseline manipulation and nondifferential test procedure as in Experiment $2 \mathrm{~A}$. In contrast to that experiment, the results of Experiment $2 \mathrm{~B}$ showed that matching accuracy was very much poorer for birds initially trained with differential food versus no-food outcomes than for birds initially trained with those outcomes scheduled nondifferentially across trials. The fact that samples alone in Group Diff were unable to support the same level of matching accuracy as they did in Group Nondiff suggests that they had been overshadowed by the expectancy of food versus no food during initial training. The reduction in conditional stimulus control by the samples was immediately evident when nondifferential tests were conducted with 0 -sec delays. It reappeared again when a memory requirement was imposed following reacquisition to criterion.

The latter result is important because it helps to rule out one other interpretation of the differences seen initially at the 0 -sec delays-namely, that the change in outcomes per se from training to testing experienced by Group Diff (but not by Group Nondiff) accounts entirely for its relatively poor test performance. If that were true, then further testing after performances had recovered to criterion levels (i.e., after the Group Diff subjects had habituated to their new outcome condition) should have revealed comparable levels of matching accuracy in the two groups. But that was not the case. Introducing mixed delays following reacquisition to criterion revealed once again that the samples alone sustained a lower level of matching accuracy in Group Diff than in Group Nondiff.

\section{EXPERIMENTS 3A AND 3B}

The last experiment in each study tested the assumption that initial differential outcomes training for the Group Diff birds in Experiments $1 \mathrm{~A}$ and $1 \mathrm{~B}$ had in fact provided those birds with a redundant outcome-expectancy cue for choice (see Table 1). An independent demonstration of this is essential to any argument that postulates that such a discriminative cue was responsible for the effects seen in the preceding experiments.

Consequently, the birds in Group Diff were returned to their differential outcomes procedure in order to reestablish the hypothesized control by outcome expectancies. Later, those outcomes were associated with new center-key stimuli outside of the matching context. Then, in a subsequent test phase, the line samples used during original training were replaced by the novel center-key stimuli such that the outcome expectancies presumably associated with the latter signaled the same matching choices as they did during prior symbolic MTS (cf. Peterson, 1984).

\section{Method}

Subjects and Apparatus. The same subjects and apparatus described previously were used in Experiments $3 \mathrm{~A}$ and $3 \mathrm{~B}$.

Procedure. After some additional nondifferential tests that proved to be uninformative, the Group Diff birds of both studies were returned to their original pretraining phase (see Experiments $1 \mathrm{~A}$ and $1 B$ ), during which the $\mathrm{V}$ and $\mathrm{H}$ center-key stimuli were reinforced with different probabilities of food (Experiment $3 \mathrm{~A}$ ) or by food versus no food (Experiment 3B). The associations between the lines and the outcomes for each bird were identical to those they had previously experienced. Differential outcomes pretraining in Experiment 3A lasted for five sessions. In Experiment 3B, this pretraining was continued for each bird until it responded differentially to the $\mathrm{V}$ and $\mathrm{H}$ lines (range: $3-5$ sessions).

The Group Diff birds of both experiments were then returned to symbolic MTS with mixed delays and with differential outcomes for the correct comparison choice following each line sample. A minimum of five mixed-delay sessions were run; birds were run until delayed-matching performances recovered to a level comparable to that exhibited at the end of Experiment 1A or 1B (range: 5-20 sessions in Experiment 3A; 5-7 sessions in Experiment 3B). Additionally, three sessions of 0-delay MTS preceded mixed-delay recovery for the birds in Experiment $3 \mathrm{~A}$.

Next, the matching procedure was again discontinued while the Group Diff birds in both experiments received off-baseline differential outcomes training with novel center-key stimuli ( $R$ and $G$ hues). Except for the change in center-key stimuli, these pretraining sessions were identical to those previously used with the $\mathrm{V}$ and $\mathrm{H}$ lines. In other words, for the birds in Experiment $3 \mathrm{~A}$, the probability of food reinforcement was 1.0 following one hue and 0.2 following the other, counterbalanced across subjects. Similarly, for the birds in Experiment 3B, food followed one center-key hue and no-food followed the other. Five sessions of off-baseline pretraining with $R$ and $G$ were given in Experiment $3 A$; three or four sessions were given in Experiment 3B.

Finally, the birds were tested on the mixed-delay procedure, with $R$ and $G$ as both samples and comparisons and with differential outcomes following correct choices on each sample trial type. The substitution of the novel $R$ and $G$ samples for the $V$ and $H$ samples used in training was done such that the outcome expectancy presumably associated with each hue sample would continue to cue the same correct choice response as it did in Experiment 1A or 1B. This meant that half of the Group Diff birds in each experiment were tested on identity MTS (i.e., the R comparison was correct following the $R$ sample, and the $G$ comparison was correct following the $G$ sample), and the other half were tested on oddity-fromsample (i.e., the $R$ comparison correct after the $G$ sample, and the $\mathrm{G}$ comparison correct after the $\mathrm{R}$ sample). All birds were tested for 10 sessions under these conditions. Prior to the first transfer test session, the birds received between one and four refresher sessions on their original, mixed-delay symbolic MTS task in order to ensure high levels of baseline accuracy.

The Group Nondiff birds in both experiments also received the same transfer test given their Group Diff counterparts. For them, however, Experiments $3 \mathrm{~A}$ and $3 \mathrm{~B}$ began immediately with $\mathrm{R}-\mathrm{G}$ differential outcomes pretraining. During transfer, half of the Group Nondiff birds were tested on identity MTS and half were tested on oddity-from-sample, with their first test session preceded by one 


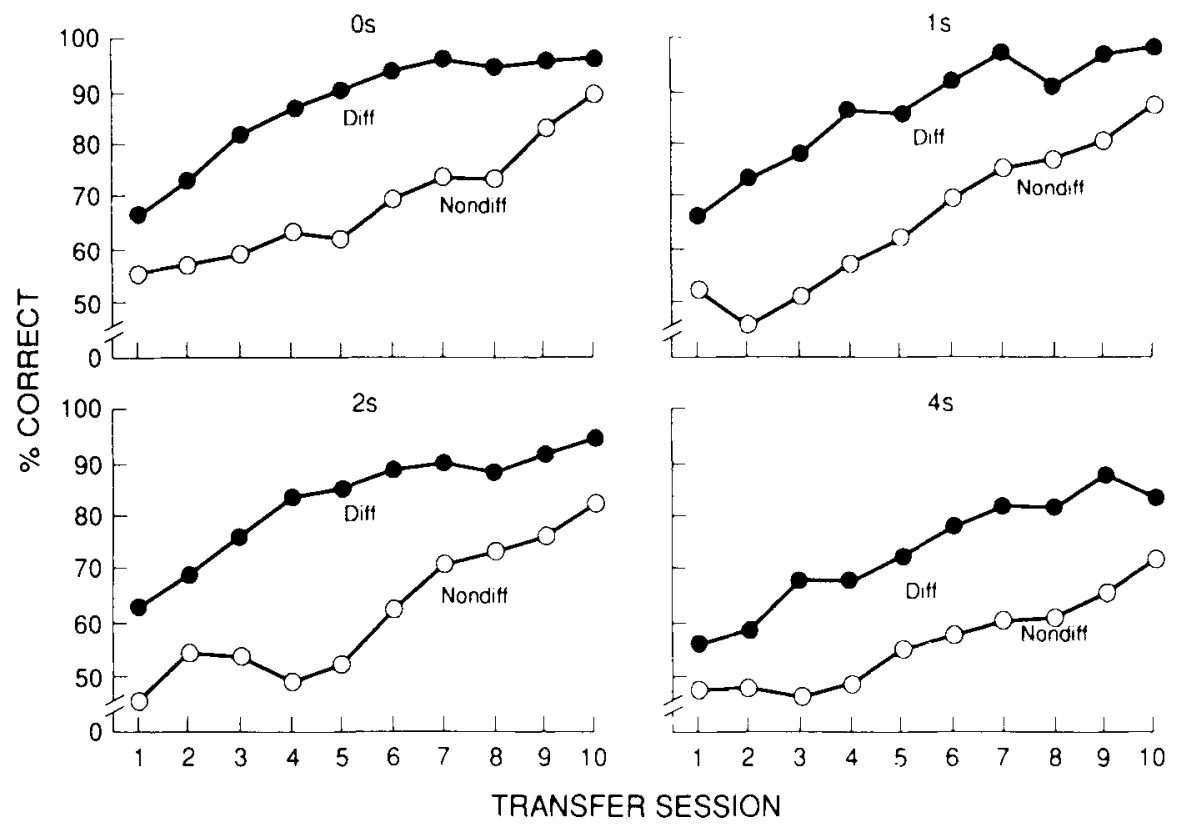

Figure 6. Acquisition by delay of the hue-hue transfer task for each group in Experiment 3A.

to three refresher sessions on their original symbolic MTS task with nondifferential outcomes. The substitution of $R$ and $G$ samples for the $\mathrm{V}$ and $\mathrm{H}$ lines in Group Nondiff was done in a fashion that, across birds, equated for the various sample-comparison test contingencies in Group Diff.

\section{Results and Discussion}

Figures 6 and 7 show the results of the transfer test in Experiments $3 \mathrm{~A}$ and $3 \mathrm{~B}$, respectively. ${ }^{2}$ Performances by the two groups in each experiment over the 10 mixed- delay sessions are plotted separately for each delay value. There are two prominent effects in each set of transfer data. First, Group Diff performed at higher levels of accuracy than did Group Nondiff at every delay. Second, matching accuracy for Group Diff in both experiments was well above chance on the very first test session (the only exception being at the 4-sec delay in Experiment 3A); corresponding accuracy for Group Nondiff was at or near chance.

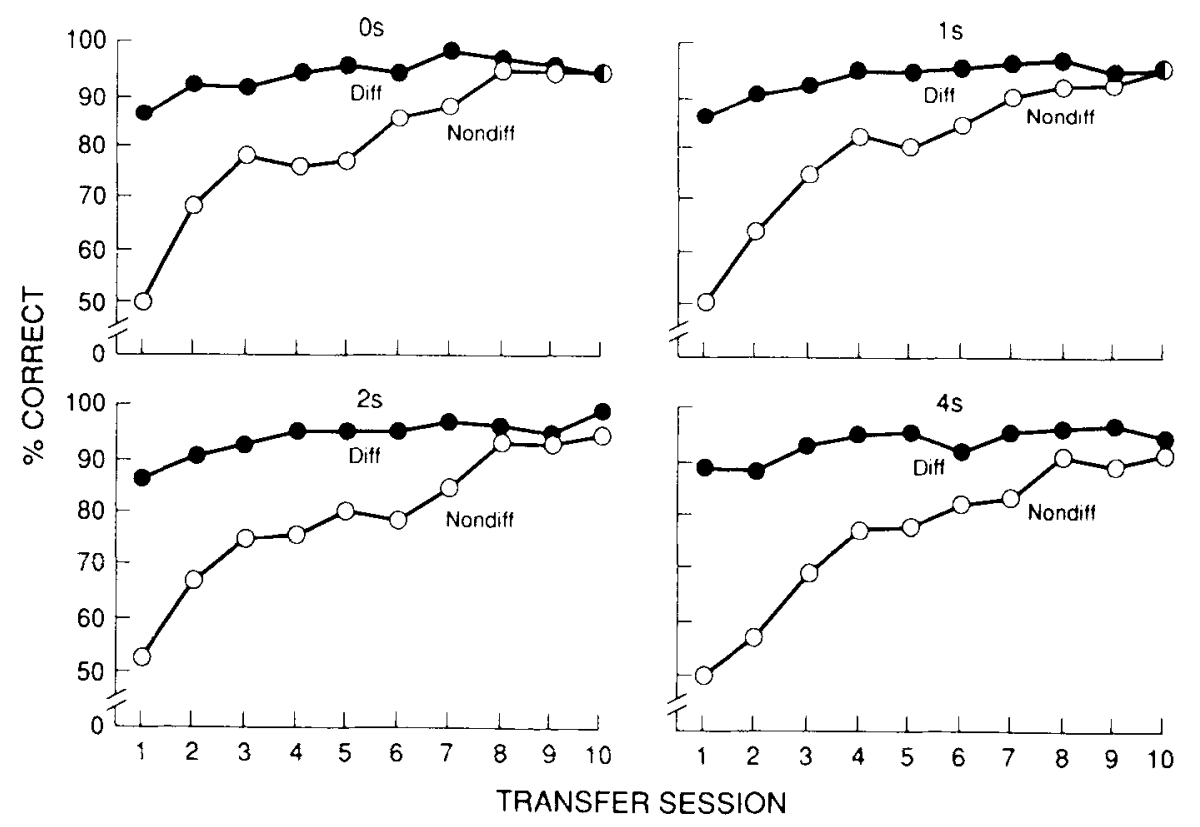

Figure 7. Acquisition by delay of the hue-hue transfer task for each group in Experiment $3 B$. 
Separate ANOVAs on the test data at each delay in Experiment 3A showed that the level of performance averaged over sessions was higher in Group Diff than in Group Nondiff at all four delays $[F(1,14)=21.43$ at the 0 -sec delay, and $F \mathrm{~s}(1,13)=24.10,24.93$, and 11.21 at the 1-, 2-, and 4-sec delays, respectively]. Also, the rate of matching reacquisition with the new sample stimuli was significantly faster in Group Diff than in Group Nondiff at all delays except $4 \mathrm{sec}$, as indicated by the group $X$ session interactions $[F(9,126)=1.84$ at the 0 -sec delay, and $F \mathrm{~s}(9,117)=1.78,2.97$, and 0.69 at the 1-, 2-, and 4-sec delays, respectively]. Finally, the effect of test session averaged across groups was significant at all delays $[F(9,126)=15.48$ at the 0 -sec delay, and $F \mathrm{~s}(9,117)=$ $25.55,22.44$, and 12.95 at the $1-, 2-$, and $4-\mathrm{sec}$ delays, respectively].

An ANOVA on the transfer data from Experiment 3B revealed a significant group effect at each delay $[F \mathrm{~s}(1,14)=24.46,24.71,17.63$, and 19.91 for the $0-$, $1-, 2-$, and 4-sec values, respectively]. The group $\times$ session interactions were also all significant $[F \mathrm{~s}(9,126)=$ $5.95,7.29,5.98$, and 6.86 , respectively]. Finally, there was a significant effect of test session averaged across groups at all four delays $[F \mathrm{~s}(9,126)=13.92,18.16$, 15.67 , and 14.90 , respectively].

These experiments show, then, that symbolic MTS performances will transfer to novel samples that have the same differential outcome associates as the samples used in training. Although the degree of positive transfer differed as a function of what those differential outcomes were, it was nonetheless evident in Group Diff of each experiment, as judged by its superior performance relative to that of the control group. This positive transfer indicates that matching by the Group Diff birds had indeed been mediated by the outcome expectancies associated with the different probabilities of food or with food versus no food in Experiments 1A and 1B, respectively.

\section{GENERAL DISCUSSION}

Two different relationships between outcome expectancies and sample stimuli are illustrated by the results of these experiments. The first is that differential outcomes can provide an additional source of conditional stimulus control in MTS that does not interfere with, or enhance, the control exerted by the samples themselves. This occurred when different nonzero probabilities of food were associated with each sample stimulus (Experiments 1A and $3 \mathrm{~A}$ ). The birds trained with these particular differential outcomes matched at the same level of accuracy as the nondifferentially trained controls when later tested with the same probability of food on all trials (Experiment 2A). Thus, the samples acquired the same degree of control over matching whether or not a redundant expectancy cue had been present during the acquisition phase.
The second relationship was demonstrated by the experiments involving food versus no food as the different outcomes (Experiments 1B and 3B). Here, the differentially trained birds matched at lower levels of accuracy than did the controls when later shifted to a test condition in which each outcome occurred equally often following all correct choices (Experiment 2B). This reduction in sample-stimulus control indicates that the redundant expectation of food versus no food during acquisition overshadowed the ability of those samples to support matching performances.

Each of these interpretations, of course, relies on the assumption that the association of different outcomes with the sample stimuli had in fact provided another discriminative cue besides the samples themselves. But this assumption was independently supported by the results of Experiments $3 \mathrm{~A}$ and $3 \mathrm{~B}$, which showed that novel stimuli readily substitute for the samples used in training provided that both sets had been associated with the same differential outcomes.

The present findings are important because of their implications for the mechanisms producing the DOE. Apparently, the DOE in conditional discrimination learning can arise because subjects have more than one cue to guide their performances and/or because one of the cues (viz., that associated with differential outcome expectancies) is especially salient. The first mechanism is suggested by the results of the experiments involving same versus different probabilities of food reinforcement. Here, the birds trained with different reinforcement probabilities acquired symbolic MTS faster and also showed better retention than did the birds trained with the same probability of food on all trials (Experiment 1A; cf. Santi \& Roberts, 1985). Later, when the samples alone provided the only cue for choice in both groups, the birds originally trained with differential outcomes were just as accurate in their choices as the birds originally trained in the nondifferential condition. By implication, then, the DOE observed during acquisition and the initial retention tests arose because the anticipation of different trial outcomes provided the differentially trained birds with an additional discriminative stimulus for performance.

By contrast, the DOE observed when the different outcomes were food versus no food (Experiment 1B) cannot be explained simply by appealing to the "summation", of control by two cues. After all, the expectation of food versus no food not only provided a redundant cue for choice but also reduced the degree to which the pigeons' matching performances were governed by the samples themselves. This suggests that the DOE in Experiment 1B arose because these differential outcome expectancies were particularly salient. Consequently, they effectively replaced the samples as the dominant cue for choice. The salience interpretation is supported by the findings that (1) the DOE was considerably larger with these outcomes than with different reinforcement probabilities and (2) transfer of performance across samples having com- 
mon differential outcome associates was much stronger with food versus no-food outcomes (see Figures 6 and 7). Indeed, there was very little loss of matching accuracy by Group Diff in Experiment 3B when R and $G$ were substituted for the $\mathrm{V}$ and $\mathrm{H}$ samples.

The overshadowing of sample-stimulus control by the expectancies of food versus no food is also consistent with the salience interpretation. Both the instrumental and Pavlovian conditioning literatures, for example, indicate that the extent to which one redundant cue reduces the discriminative control by another is affected by their relative discriminability (e.g., Lovejoy \& Russell, 1967; Mackintosh, 1976; Miles \& Jenkins, 1973). If the size of the DOE and the degree of sample substitutability are legitimate indices of this property of outcome expectancies, then the cue associated with food versus no-food outcomes would be expected to yield a relatively large reduction in conditional stimulus control by the samples. Experiment $2 \mathrm{~B}$ confirmed this prediction by demonstrating an overshadowing effect that, until now, had only been suggested in the differential outcomes literature (e.g., Honig et al., 1984).

The results of Experiment $2 \mathrm{~A}$ are also in line with this prediction given that the expectation of different probabilities of food, which produced a smaller DOE and weaker transfer effects, did not appear to reduce samplestimulus control over matching performance. But did the relatively weak expectancy cue associated with those outcomes really have no effect on the samples? Or were real effects (e.g., overshadowing) simply not detected because the nondifferential test procedure was insensitive to them?

The possibility that an insensitive test procedure was responsible for the findings of Experiment $2 \mathrm{~A}$ seems unlikely given that the same general test procedure did detect differences in sample-stimulus control as a function of differential versus nondifferential outcomes training in Experiment 2B. A more plausible alternative, however, is that the degree of control acquired by the samples in Experiment $1 \mathrm{~A}$ was affected by how strongly outcome expectancies controlled matching performances but that this effect was masked because the results of the Group Diff birds were not homogeneous. For example, the samples alone may have been unable to support high levels of matching accuracy for the birds whose outcome expectancies exerted relatively strong control over choice. Conversely, the samples may have been the dominant cue for the birds whose expectancy cue was relatively weak. If so, averaging the Group Diff data together in Experiment $2 \mathrm{~A}$ could have obscured an overshadowing effect in some birds.

This hypothesis clearly predicts a negative correlation between sample-stimulus control and control by outcome expectancies. In other words, the degree of transfer observed for the Group Diff birds in Experiment 3A (where control by outcome expectancies was assessed) should be inversely proportional to the level of their nondifferential test performances in Experiment $2 \mathrm{~A}$, which assessed sample-stimulus control. Table 2 summarizes some data relevant to this prediction. The average performance for
Table 2

Percentages of Correct Choice Responses for the Group Diff Birds in Experiments 2A and 3A Averaged over Delays for the 10 Mixed-Delay Sessions with Nondifferential Reinforcement (Experiment 2A), the First Transfer Session (Experiment 3A), and for all 10 Transfer Sessions

\begin{tabular}{|c|c|c|c|}
\hline \multirow{2}{*}{$\begin{array}{l}\text { Diff } \\
\text { Bird }\end{array}$} & \multirow{2}{*}{$\begin{array}{l}\text { Average Nondiff } \\
\text { Mixed Delays }\end{array}$} & \multicolumn{2}{|c|}{ Transfer } \\
\hline & & First Session & Average \\
\hline$\overline{D F} 1$ & 61.0 & 65.0 & 82.9 \\
\hline DF2 & 72.4 & 45.0 & 73.0 \\
\hline DF3 & 65.2 & 51.2 & 83.0 \\
\hline DF5 & 72.9 & 83.8 & 92.1 \\
\hline DF6 & 80.0 & 72.5 & 85.5 \\
\hline DF7 & 91.5 & 60.0 & 84.9 \\
\hline DF8 & 65.2 & 60.0 & 77.0 \\
\hline
\end{tabular}

7 of the 8 Group Diff birds on the mixed-delay tests of Experiment $2 \mathrm{~A}$ are shown as an index of control by the samples alone (see Note 2); accuracy averaged over all delays on the first session of transfer and across all transfer sessions are presented as indices of control by outcome expectancies. The correlations between nondifferential performance and transfer performances were .13 and .27, respectively. Thus, there is no evidence here to support the argument that averaging the Group Diff data together in Experiment 2A masked a true overshadowing effect.

As mentioned above, the difference in results obtained with the two outcome manipulations suggests that the salience of the expectancy cue was an important factor in obtaining an overshadowing effect. However, it is also necessary to consider an explanation in terms of the presence versus absence of differential sample responding (see Urcuioli, 1984). For instance, the Group Diff birds in Experiment 1B pecked the food samples more often and with a shorter latency than the no-food samples, whereas the Group Diff birds in Experiment 1A responded rather similarly to the two samples. Considering that Urcuioli (1984) found that differential sample behaviors reduce (overshadow) the samples for control over choice, might this also be responsible for the effect seen in Experiment 2B (and also help to explain its absence in Experiment 2A)?

If differential sample responding truly provided the overshadowing cue for the Group Diff birds during initial training in Experiment 1B, then matching by those birds could be described by "if, then" performance rules, such as "if responding to the sample, then peck the $R$ comparison" and "if not responding to the sample, then peck the G comparison"' (see Carter \& Werner, 1978). By implication, "responding to the sample"' ought to induce a strong bias to peck the comparison cued by that particular response pattern during nondifferential testing, because the shift to nondifferential outcomes (including the off-baseline phase) generated considerable responding to both samples. Contrary to this prediction, however, the percentage of choice responses occurring to the comparison previously associated with sample responding in Group Diff in Experiment $2 \mathrm{~A}$ averaged only $54 \%$ over the first three nondifferential test sessions in Experi- 
ment 2B. The corresponding figure for the first five mixed-delay sessions that followed was $51 \%$. Clearly, the relatively poor performance of the Group Diff birds in Experiment 2B cannot be explained simply in terms of a bias to peck one comparison in preference to the other. Consequently, the outcome-expectancy cue in this study cannot simply be "reduced" to different patterns of sample responding.

Although the precise nature of the outcome-expectancy cues in these experiments may be unclear, it is most certainly clear that the presence versus absence of food reinforcement, and the different probabilities of food, generated an additional cue for choice responding for the Group Diff birds. More importantly, when that cue was particularly salient, it produced an effect very much like that seen in other redundant-cue paradigms: It overshadowed the control normally acquired by its partner.

\section{REFERENCES}

Brodigan, D. L., \& Peterson, G. B. (1976). Two-choice conditional discrimination performance of pigeons as a function of reward, expectancy, prechoice delay, and domesticity. Animal Learning \& Behavior, 4, 121-124.

Carlson, J. G., \& Wielkiewicz, R. M. (1976). Mediators of the effects of magnitude of reinforcement. Learning \& Motivation, 7, 184-196.

Carter, D. E., \& Werner, T. J. (1978). Complex learning and information processing by pigeons: A critical analysis. Journal of the $E x-$ perimental Analysis of Behavior, 29, 565-601.

Cohen, L. R., Looney, T. A., Brady, J. H., \& Aucella, A. F. (1976). Differential sample response schedules in the acquisition of conditional discriminations by pigeons. Journal of the Experimental Analysis of Behavior, 26, 301-316.

DeLong, R. E., \& Wasserman, E. A. (1981). Effects of differential reinforcement expectancies on successive matching-to-sample performance by pigeons. Journal of Experimental Psychology: Animal Behavior Processes, 7, 394-412.

Edwards, C. A., Jagielo, J. A., Zentall, T. R., \& Hogan, D. E. (1982). Acquired equivalence and distinctiveness in matching to sample by pigeons: Mediation by reinforcer-specific expectancies. Journal of Experimental Psychology: Animal Behavior Processes, 8, 244-259.

HoNIG, W. K., \& DodD, P. W. D. (1986). Anticipation and intention in working memory. In D. F. Kendrick, M. E. Rilling, \& M. R. Denny (Eds.), Theories of animal memory (pp. 77-100). Hillsdale, NJ: Erlbaum.

Honig, W. K., Matheson, W. R., \& Dodd, P. W. D. (1984). Outcome expectancies as mediators for discriminative responding. Canadian Journal of Psychology, 38, 196-217.

Lovejoy, E., \& Russell, D. G. (1967). Suppression of learning about a hard cue by the presence of an easy cue. Psychonomic Science, 8 , 365-366.

MaCKINTOSH, N. J. (1974). The psychology of animal learning. New York: Academic Press

Mackintosh, N. J. (1976), Overshadowing and stimulus intensity. Animal Leaming \& Behavior, 4, 186-192.

Miles, C. G., Jenkins, H. M. (1973). Overshadowing in operant conditioning as a function of discriminability. Learning \& Motivation, 4, 11-27.
Peterson, G. B. (1984). How expectancies guide behavior. In H. L. Roitblat, T. G. Bever, \& H. S. Terrace (Eds.), Animal cognition (pp. 135-147). Hillsdale, NJ: Erlbaum.

Peterson, G. B., \& Trapold, M. A. (1980). Effects of altering outcome expectancies on pigeons' delayed conditional discrimination performance. Learning \& Motivation, 11, 267-288.

Peterson, G. B., \& Trapold, M. A. (1982). Expectancy mediation of concurrent conditional discriminations. American Journal of Psychology, 95, 571-580.

Peterson, G. B., Wheeler, R. L., \& Armstrong, G. D. (1978). Expectancies as mediators in the differential-reward conditional discrimination performance of pigeons. Animal Learning \& Behavior, 6, 279-285

Peterson, G. B., Wheeler, R. L., \& Trapold, M. A. (1980). Enhancement of pigeons' conditional discrimination performance by expectancies of reinforcement and nonreinforcement. Animal Learning \& Behavior, 8, 22-30.

RODGER, R. S. (1975). The number of non-zero, post-hoc contrasts from ANOVA and error rate. I. British Journal of Mathematical and Statistical Psychology, 28, 71-78.

SANTI, A., \& RoBerTs, W. A. (1985). Reinforcement expectancy and trial spacing effects in delayed matching-to-sample by pigeons. Animal Learning \& Behavior, 13, 274-284.

Trapold, M. A. (1970). Are expectancies based upon different positive reinforcing events discriminably different? Learning \& Motivation, 1, 129-140.

URCUIOLI, P. J. (1984). Overshadowing in matching-to-sample: Reduction in sample-stimulus control by differential sample behaviors. Animal Learning \& Behavior, 12, 256-264.

URCUIOLI, P. J. (1985). On the role of differential sample behaviors in matching-to-sample. Journal of Experimental Psychology: Animal Behavior Processes, 4, 502-519.

UrCuioli, P. J., \& Honig, W. K. (1980). Control of choice in conditional discriminations by sample-specific behaviors. Journal of Experimental Psychology: Animal Behavior Processes, 6, 251-277.

\section{NOTES}

1. The retention function for each group in Experiment 1A was computed on the basis of the data from only 7 subjects. The remaining subjects were excluded because they responded rapidly to one of the dark side keys during all delays longer than $0 \mathrm{sec}$, thus precluding an accurate assessment of retention. The Group Nondiff retention function in Experiment 1B also excludes the data from 1 subject for the same reason. Also, since this behavior persisted for these same subjects in Experiments $2 \mathrm{~A}$ and $2 \mathrm{~B}$, their data were also not included in the group retention functions for those experiments.

2. All 8 subjects in each group in Experiment $3 A$ were included in the averaged transfer functions at the 0 -sec delay. For the longer delays, 1 subject in Group Diff was omitted from the analysis because it continued to respond rapidly to one side key during the delays over the first five test sessions. However, the 1 corresponding subject in Group Nondiff that had been omitted from the retention-data analyses in Experiments $1 \mathrm{~A}$ and $2 \mathrm{~A}$ was now included because it no longer engaged in that same sort of delay-interval behavior. Consequently, group sizes in the analysis of Experiment $3 \mathrm{~A}$ were 7 and 8 for delays longer than $0 \mathrm{sec}$. All 8 subjects in each group were included in the transfer functions for Experiment 3B.

(Manuscript received October 5, 1989; revision accepted for publication March 23, 1990.) 